\title{
Development of an assay to assess the functional integrity of the human sperm membrane and its relationship to other semen characteristics
}

\author{
R. S. Jeyendran* $\uparrow$, H. H. Van der Ven $\uparrow \S$, M. Perez-Pelaez*, B. G. Crabo $\ddagger$ \\ and L. J. D. Zaneveld $†$ T
}

*Institute of Reproductive Medicine and Infertility, Chicago, Illinois 60602; $\dagger$ Departments of Physiology and Biophysics, and Obstetrics and Gynecology, University of Illinois at the Medical Center, Chicago, Illinois 60612; and $\ddagger$ Department of Animal Science, University of Minnesota, St Paul, Minnesota 55108, U.S.A.

\begin{abstract}
Summary. The objective of this study was to develop a relatively simple test to evaluate the functional integrity of the membranes of human spermatozoa. As in some other species, human spermatozoa 'swell' under hypo-osmotic conditions due to the influx of water and the expansion of the membranes. A mixture of equal parts of fructose and sodium citrate (150 mosmol) with calculated ionic strength of 0.15 resulted in a maximal number of clearly identifiable swollen spermatozoa. Only small variations were seen when different aliquants of the same semen samples were separately evaluated. A high correlation $(r=0.94)$ was obtained between expected and observed values of swollen spermatozoa when known amounts of heat-treated spermatozoa, unable to undergo swelling, were added to untreated spermatozoa. A good correlation $(r=0.90)$ was also observed between the $\%$ spermatozoa in a semen sample that were capable of undergoing swelling and the \% of denuded hamster oocytes that were penetrated by capacitated spermatozoa from the same semen sample. By contrast, the correlations between $\%$ sperm swelling in ejaculates and $\%$ normal sperm forms, $\%$ motile spermatozoa and \% spermatozoa that do not stain with eosin-Y (supravital stain) in the same ejaculates were $0 \cdot 30,0 \cdot 61$ and 0.52 , respectively. Therefore, the hypoosmotic swelling technique to evaluate the functional integrity of the sperm membrane appears to give high repeatability and accuracy and is closely correlated to the in-vitro fertilizing ability of spermatozoa. It may be a useful addition to the standard semen analysis.
\end{abstract}

\section{Introduction}

In the diagnosis of male infertility, a number of sperm characteristics are evaluated. However, only limited attention has been given to assessing the functional integrity of the sperm membrane. Membrane integrity is not only important for sperm metabolism, but a correct change in the properties of the membrane is required for successful union of the male and female gametes, i.e. for sperm capacitation, the acrosome reaction, and the binding of the spermatozoon to the egg surface.

$\S$ Present address: Medizinische Hochschule Luebeck, Klinik für Frauenheilkunde, D-2400 Luebeck 1, West Germany.

I Present address: Rush-Presbyterian St Luke's Medical Center, 1753 W. Congress Parkway, Chicago, Illinois 60612 , U.S.A. 
Thus, the integrity and functional activity of the sperm membrane is of fundamental importance in the fertilization process, and assessment of membrane function may be a useful indicator of the fertilizing ability of spermatozoa.

Presently, no simple test is available for this purpose. Histological studies only show whether the membrane is morphologically intact. The same is true for the 'live-dead' ('supravital') stain which measures the ability of eosin-Y dye to pass through membranes, a sign that the membranes are morphologically altered due to cell injury or death. Therefore, it would be worthwhile to develop a technique that measures the functional integrity of the sperm membrane and that can be readily applied clinically.

A property of the cell membrane is its ability to permit the transport of molecules selectively. When exposed to hypo-osmotic conditions, water will enter the spermatozoon in an attempt to reach osmotic equilibrium. This inflow of water will increase sperm volume and the plasma membrane will bulge (balloon), giving minimum surface to volume ratio. The sperm tail appears to be particularly susceptible to such hypo-osmotic conditions. These induced alterations in sperm morphology are visible with the phase-contrast microscope. For simplicity, this ballooning effect will be referred to as 'swelling'. Drevius \& Eriksson (1966) observed the swelling phenomenon of the sperm tail when bovine spermatozoa were exposed to a hypo-osmotic $(0.024$ to $0.043 \mathrm{M}$-sodium chloride) solution. Since then, several studies have confirmed the ability of bovine spermatozoa to swell in a hypo-osmotic medium (Bredderman \& Foote, 1969; Foote \& Bredderman, 1969; Drevius, 1972).

It can be assumed that the ability of the sperm tail to swell in the presence of a hypo-osmotic solution is a sign that transport of water across the membrane occurs normally, i.e. is a sign of membrane integrity and normal functional activity. This may be a useful observation when performing a semen analysis in the diagnosis of the infertile male. The objectives of the present study were: (1) to evaluate whether human spermatozoa also swell in a hypo-osmotic solution; (2) to determine the solution in which a clearly identifiable swelling of a maximum number of spermatozoa occurs; and (3) to correlate the ability of the spermatozoa to swell in this hypoosmotic solution with different sperm variables as well as with their ability to undergo capacitation and penetrate oocytes.

\section{Materials and Methods}

Assay technique. Ejaculates were obtained from men by self-masturbation. Semen samples from men known to be of infertile marriages were used to determine the relationship between sperm swelling, sperm morphology, sperm motility and 'live' spermatozoa, whereas all other experiments were performed with spermatozoa from apparently normal, healthy men who had either fathered children or had never attempted to procreate, and whose ejaculates appeared normal in all aspects (Zaneveld \& Polakoski, 1977). Very rarely, semen specimens contained more than $5 \%$ swollen spermatozoa or coiled tails; these were rejected. The semen samples were used within $60 \mathrm{~min}$ of complete liquefaction. If liquefaction required more than $30 \mathrm{~min}$, the sample was rejected. Pooled ejaculates were used to select the optimal hypo-osmotic solution. Individual semen samples were employed to determine the repeatability and accuracy of the assay techniques, and to evaluate the relationship between the incidence of sperm swelling under hypo-osmotic stress and the other semen characteristics as well as with the ability of the spermatozoa from the same semen sample to become capacitated in an artificial medium and to penetrate denuded hamster oocytes.

The osmotic swelling technique consisted of mixing $0.1 \mathrm{ml}$ whole semen with $1.0 \mathrm{ml}$ hypoosmotic solution and allowing the mixture to incubate at $37^{\circ} \mathrm{C}$ for at least $30 \mathrm{~min}$. A modified $(1.5$ mm thick) Petroff Hausser Chamber with a cell depth of $0.02 \mathrm{~mm}$ and $1 / 400 \mathrm{~mm}^{2}$ ruling (Hausser Scientific, Philadelphia, Pennsylvania) was filled with the sperm mixture. The mixture was allowed to stand for at least $1 \mathrm{~min}$ before observations were made by phase-contrast microscopy at $\times 250$ 
(without staining); 100 to 112 spermatozoa were observed in 5 non-touching squares of the chamber. The percentage spermatozoa that showed typical tail abnormalities indicative of swelling was calculated (number of altered spermatozoa $\times 100$, divided by total number of spermatozoa counted in the same area).

Selection of the hypo-osmotic solution. Since sugars and electrolytes are known to maintain the functional integrity of spermatozoa, solutions were made of three sugars with different molecular weights (fructose, melitose and sucrose) as well as two electrolytes (sodium citrate and sodium chloride) in water and their ability to induce sperm swelling was evaluated. The osmolality of the solutions was varied from 0 to $300 \mathrm{mosmol}$, at $50 \mathrm{mosmol}$ increments as determined by freezing point depression (Advanced Digimatic Osmometer, Model 3D11, American Sci. Prod., Chicago, Illinois).

Semen samples from 3 donors were pooled, mixed thoroughly and divided into 35 fractions. Each portion was incubated with one of the solutions and the percentage spermatozoa with swollen sperm tails was determined. After the optimal osmolarity, sugar and electrolyte values were established, the effect of varying the ionic strength on sperm swelling was studied by retaining the osmolarity of the solution but varying the ratio of sugar and electrolyte.

Repeatability and accuracy of the technique. After having determined the optimal hypo-osmotic solution (fructose and sodium citrate at $150 \mathrm{mosmol}$ and a calculated ionic strength of 0.15 ; see 'Results'), the repeatability of the assay technique was established by dividing a semen sample into 12-15 equal portions. Each portion was treated with the hypo-osmotic solution as described above and the percentage spermatozoa showing alterations was determined. For this purpose, semen specimens were selected which had been shown to vary largely in the percentage of spermatozoa that underwent swelling in a hypo-osmotic solution.

When spermatozoa are subjected to moderate heat $\left(56^{\circ} \mathrm{C}\right)$ for $30 \mathrm{~min}$, they undergo membrane changes and swelling of the sperm tail generally does not occur when exposed to hypo-osmotic stress. To determine the reliability of the swelling test response in relation to the number of spermatozoa that are capable of undergoing swelling, aliquants of untreated fresh semen samples were mixed with semen that had been heat-treated. Various proportions were studied so that the mixture contained $100,75,50,25$ or $10 \%$ heat-treated spermatozoa. These mixtures were subsequently subjected to the same hypo-osmotic conditions as described previously.

Relationship of sperm swelling to in-vitro penetration of denuded hamster oocytes, sperm motility, sperm morphology and the live-dead (supravital) stain. Semen samples from 23 different men were each divided into two aliquots. These men were either known to be fertile and/or possessed normal ejaculates (see above). In one of the aliquots, the percentage of spermatozoa showing swelling under hypo-osmotic conditions was assessed. In the other, the ability of the spermatozoa to fuse with oocytes was determined by incubating them with denuded hamster oocytes (Rogers et al., 1979; Binor, Sokoloski \& Wolf, 1980; Van der Ven, Bhattacharyya, Binor, Leto \& Zaneveld, 1982). Spermatozoa were washed by addition of modified BWW medium containing $35 \mathrm{mg}$ human serum albumin/ml (culture medium) (Overstreet, Yanagimachi, Katz, Hayashi \& Hanson, 1980), and by centrifugation at $500 \mathrm{~g}$ for $3 \mathrm{~min}$. This procedure was repeated twice more and the spermatozoa were capacitated by incubation in culture medium for $2-3 \mathrm{~h}$ at $37^{\circ} \mathrm{C}$. The spermatozoa $(0.02 \mathrm{ml}, 2$ $\times 10^{6}$ ) were then mixed with hamster oocytes (denuded by treatment with hyaluronidase and trypsin) in $0.2 \mathrm{ml}$ culture medium and the sperm-oocyte mixture was incubated for $5 \mathrm{~h}$. The oocytes were then removed, washed, fixed, stained with aceto-lacmoid, and examined microscopically for sperm penetration. Oocytes were considered penetrated when a swollen sperm head or a male pronucleus with a corresponding sperm tail was found within the oocyte.

Semen specimens from 40 different male spouses of infertile marriages were divided into 2 aliquots. One of the aliquots was subjected to a standard semen analysis (Zaneveld \& Polakoski, 1977) to determine sperm motility and morphology (both head and tail abnormalities). Sperm motility was expressed as the \% spermatozoa that showed forward progression rather than the overall $\%$ motility (spermatozoa with and without forward progression) because the former 
probably reflects the fertilizing capacity of a semen sample more closely than the latter. The proportion of spermatozoa that showed swelling in the hypo-osmotic solution was evaluated in the other aliquot.

Similarly, 38 semen samples from different males of infertile marriages were each divided into 2 aliquots. In one of the aliquots, the percentage of spermatozoa that showed swelling in the hypoosmotic solution was determined and in the other, the percentage of spermatozoa that did not stain with eosin-Y (Eliasson \& Treichl, 1971) was evaluated.

Statistical analysis. The mean and standard deviation, and, when appropriate, Pearson's coefficient of correlation, were calculated. The statistical significance was determined from tables.

\section{Results}

\section{Morphological changes}

Human spermatozoa showed typical morphological changes when subjected to a hypo-osmotic solution (Text-fig. 1, Pl. 1, Fig. 1). These changes were clearly visible by phase-contrast microscopy. All reacting spermatozoa showed swelling within the first $30 \mathrm{~min}$. The initial response to the hypo-osmotic solution was a small enlargement of the tip of the tail or at the junction of the midpiece and tailpiece. Later, the alterations were characterized by the presence of a swollen area at the tip of the tail (Text-fig. 1b-d), a hairpin curvature of the tail (Text-fig. 1c-e), a shortened and thickened tail (Text-fig. 1f), or a swollen area that partly or completely enveloped the curved tail of the spermatozoon (Text-fig. 1d, e \& g). The incubation time did not appear to be important beyond $30 \mathrm{~min}$ : after this time, no further increase in the number of swollen spermatozoa occurred, although longer incubation resulted in swelling which was easier to identify. Occasionally, some swollen spermatozoa were also visible in untreated semen samples, but these were hardly ever more than $3-5 \%$.

The proportion of spermatozoa that showed swelling varied with the solutions used even at the same osmolality (Table 1). The differences were greatest at higher osmolality. Maximal swelling occurred in most of the solutions at 150 mosmol or less. Essentially, no differences between the electrolyte solutions were noted (Table 1). However, the sperm swelling was more readily identifiable in sodium citrate (Text-fig. Id, e \& g) than in sodium chloride (Text-fig. 1f). The fructose solution at $150 \mathrm{mosmol}$ consistently caused the highest percentage of swollen sperm tails and this sugar was used further at that osmolality. Since sperm swelling was somewhat difficult to see in this solution, sodium citrate was added at the same osmolality.

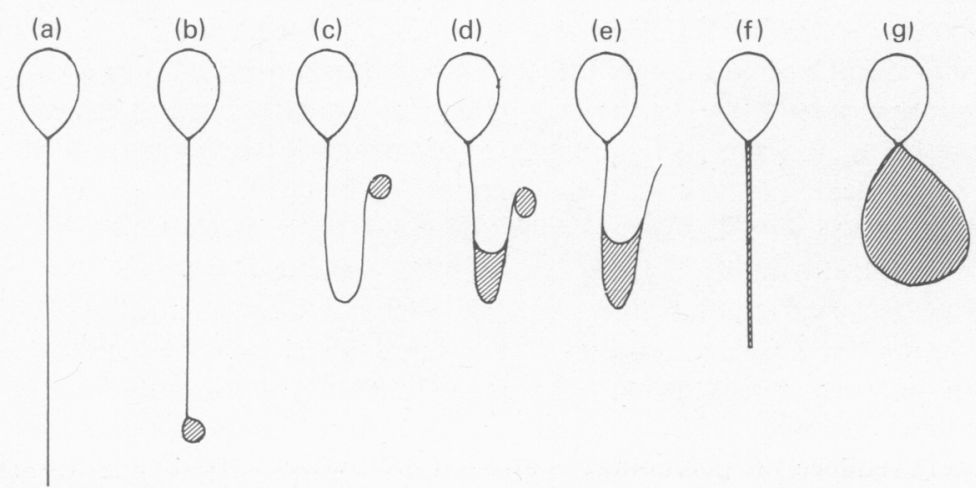

Text-fig. 1. Schematic representation of typical morphological changes of human spermatozoa subjected to hypo-osmotic stress: $a=$ no change; $b-g=$ various types of tail changes. Tail region showing swelling is indicated by the hatched area. 
PLATE 1


Fig. 1. Different types of swelling (see Text-fig. 1) of human spermatozoa in hypo-osmotic conditions.

Fig. 2. Human spermatozoa all showing type g swelling.

(Facing p. 222) 
Table 1. Effect of different solutions and varied osmolality on the swelling of human spermatozoa*

\begin{tabular}{|c|c|c|c|c|c|}
\hline \multirow{2}{*}{$\begin{array}{l}\text { Osmotic pressure } \\
\text { (mosmol) }\end{array}$} & \multicolumn{5}{|c|}{$\%$ spermatozoa swollen (mean \pm s.d.) } \\
\hline & Melitose & Sucrose & Fructose & Sodium citrate & $\mathrm{NaCl}$ \\
\hline 0 & $47 \cdot 6 \pm 2 \cdot 3$ & $44.4 \pm 2.5$ & $47.5 \pm 1.6$ & $47 \cdot 5 \pm 2 \cdot 6$ & $43 \cdot 6 \pm 2 \cdot 4$ \\
\hline 50 & $42.9+3.7$ & $41.7+3.8$ & $41.9+4.7$ & $51.7+9.6$ & $52.6+4.0$ \\
\hline 100 & $43.8 \pm 4.3$ & $39.4 \pm 2.9$ & $44.4 \pm 2.5$ & $44.4 \pm 3.7$ & $53.1 \pm 4.9$ \\
\hline 150 & $36 \cdot 1+4 \cdot 4$ & $43 \cdot 2+2 \cdot 2$ & $60 \cdot 0+6.2$ & $45.0 \pm 2.9$ & $54.3 \pm 4.0$ \\
\hline 200 & $22.3 \pm 3.8$ & $46.8 \pm 1.8$ & $58 \cdot 3 \pm 6 \cdot 3$ & $30 \cdot 0 \pm 4 \cdot 7$ & $25 \cdot 0 \pm 2.7$ \\
\hline 250 & $20.0 \pm 4.4$ & $41.4 \pm 3.1$ & $47.1 \pm 2.1$ & $25.0 \pm 5.7$ & $28.1 \pm 10.4$ \\
\hline 300 & $5.6 \pm 2 \cdot 1$ & $31.8 \pm 6.1$ & $34.1 \pm 4.0$ & $15.5 \pm 2.9$ & $12.3 \pm 3.9$ \\
\hline
\end{tabular}

* Pooled semen samples were divided into 35 different aliquots and each sample was incubated with the indicated solutions at $37^{\circ} \mathrm{C}$ for $30 \mathrm{~min}$. See text for additional detail. The test was repeated 3 times with different pooled semen samples.

Table 2. Effect of varied ionic strength of the swelling of human spermatozoa

\begin{tabular}{cccc}
\hline $\begin{array}{c}\text { Fructose } \\
(150 \text { mosmol })\end{array}$ & $\begin{array}{c}\text { Sodium citrate } \\
(150 \text { mosmol })\end{array}$ & $\begin{array}{c}\text { Calculated } \\
\text { ionic strength* }\end{array}$ & $\begin{array}{c}\% \text { Sperm } \\
\text { swollen** }\end{array}$ \\
\hline $100 \%$ & $0 \%$ & 0.00 & $51 \cdot 7 \pm 11 \cdot 8$ \\
$75 \%$ & $25 \%$ & 0.08 & $53 \cdot 5 \pm 14 \cdot 3$ \\
$50 \%$ & $50 \%$ & $0 \cdot 15$ & $60 \cdot 1 \pm 12 \cdot 5$ \\
$25 \%$ & $75 \%$ & $0 \cdot 23$ & $50 \cdot 9 \pm 15 \cdot 0$ \\
$0 \%$ & $100 \%$ & 0.30 & $50 \cdot 3 \pm 15 \cdot 3$ \\
\hline
\end{tabular}

* Assuming complete dissociation.

** Mean \pm s.d. for 6 observations. See text for experimental detail.

Only small differences were observed when the calculated ionic strength of the fructose and sodium citrate mixtures were varied from 0 to 0.30 by altering the fructose and sodium citrate concentrations while keeping the osmolality at 150 mosmol (Table 2). However, a mixture of equal volumes of fructose and sodium citrate with ionic strength of $0 \cdot 15$ consistently gave the highest percentage of swollen spermatozoa. This mixture was therefore selected as the test solution for all other experiments. To prepare this solution, $7 \cdot 35 \mathrm{~g}$ sodium citrate $2 \mathrm{H}_{2} \mathrm{O}$ and $13.51 \mathrm{~g}$ fructose are mixed with $1000 \mathrm{ml}$ distilled $\mathrm{H}_{2} \mathrm{O}$. Subsequently, $1 \mathrm{ml}$ is added to $0.1 \mathrm{ml}$ of semen and the spermatozoa are observed after a $30 \mathrm{~min}$ incubation period.

\section{Reliability of response to hypo-osmotic conditions}

Reasonably consistent results were obtained when different samples of the same ejaculate were tested (Table 3). The consistency appeared to increase with an increased percentage of swollen spermatozoa. The variability that is seen can be attributed to error of observation which occurs whenever semen characteristics are assessed. Also, no differences were noted when instead of $100-$ 112 spermatozoa, up to 200 spermatozoa were evaluated in the counting chamber and the $\%$ swollen spermatozoa was calculated.

Heat treatment reduced the percentage of swollen spermatozoa from $73 \pm 1 \cdot 2 \%$ to $8 \cdot 8 \pm 3 \cdot 2 \%$ (mean \pm s.d.) (Text-fig. 2). Dilution of untreated spermatozoa with heat-treated spermatozoa caused a linear decrease in percentage of swelling (correlation coefficient between the expected and observed sperm swelling was 0.94 ; Text-fig. 2). This demonstrates the reliability of the assay and 
Table 3. Repeatability of the assay*

\begin{tabular}{ccccc}
\hline & & \multicolumn{3}{c}{$\%$ spermatozoa swollen } \\
\cline { 3 - 5 } Ejaculate* $^{*} \begin{array}{c}\text { No. of } \\
\text { samples tested }\end{array}$ & Mean \pm s.d. & Range & $\begin{array}{c}\text { Coefficient of } \\
\text { variation }\end{array}$ \\
\hline 1 & 15 & $33 \cdot 8 \pm 3 \cdot 0$ & $31-41$ & 0.09 \\
2 & 12 & $35 \cdot 3 \pm 5 \cdot 5$ & $27-43$ & $0 \cdot 16$ \\
3 & 15 & $65 \cdot 2 \pm 4 \cdot 2$ & $59-74$ & 0.06 \\
4 & 12 & $80.9 \pm 2 \cdot 6$ & $77-86$ & 0.03 \\
\hline
\end{tabular}

* The ejaculates were obtained from different donors and divided into a number of samples. Each sample was treated with the hypo-osmotic solution as described in the text and the percentage of swollen spermatozoa determined.

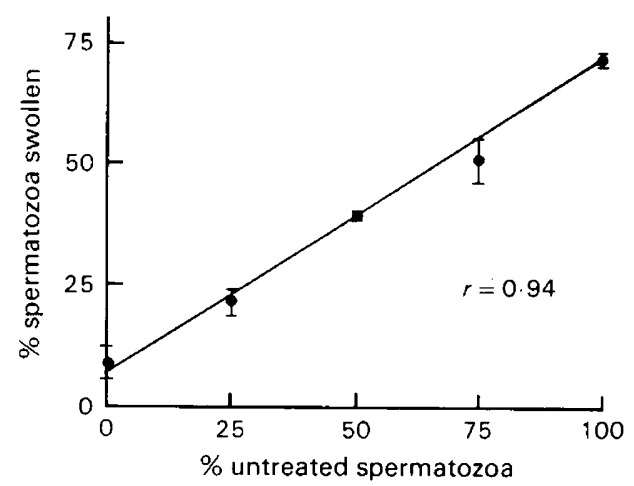

Text-fig. 2. Relationship between the $\%$ spermatozoa in a sample subjected to heat treatment $\left(56^{\circ} \mathrm{C}, 30 \mathrm{~min}\right)$ and the \% of spermatozoa that swell under hypo-osmotic conditions. Values are mean \pm s.d. for 12 observations. All individual datum points were used to calculate the correlation coefficient. See text for experimental detail.

also that the number of spermatozoa that show swelling is not influenced by the number of spermatozoa unable to swell.

\section{Correlation of sperm swelling with various sperm characteristics}

A good correlation $(r=0.90, P<0.001)$ existed between the percentage of spermatozoa that showed swelling under hypo-osmotic conditions in a semen specimen and the ability of spermatozoa from that sample to undergo capacitation and penetrate oocytes (Text-fig. 3a). Overall, the percentage of swollen spermatozoa (mean \pm s.d.) in the ejaculates was $62 \cdot 2 \pm 18 \cdot 1 \%$ and the percentage of oocyte penetration was $65.6 \pm 28.6 \%(n=23)$. Ejaculates that showed an oocyte penetration rate of $>55 \%$ produced a higher correlation with sperm swelling than did those with a penetration rate of $<55 \%$. Even so, with the exception of one ejaculate, each ejaculate that showed a penetration rate of $<55 \%$ also showed $<55 \%$ swollen spermatozoa.

By contrast, very little relationship existed between the percentage of swollen spermatozoa and the percentage of normal sperm forms present in the ejaculate $(r=0 \cdot 30)$ (Text-fig. 3b). Higher correlations were found between the percentage of motile and swollen spermatozoa $(r=0.61 ; P<$ 0.01 ) and between the percentage of swollen spermatozoa and the spermatozoa that did not stain with eosin-Y $(r=0.52 ; P<0.01)$ (Text-fig. 3c, d). In contrast to the percentage of oocyte 


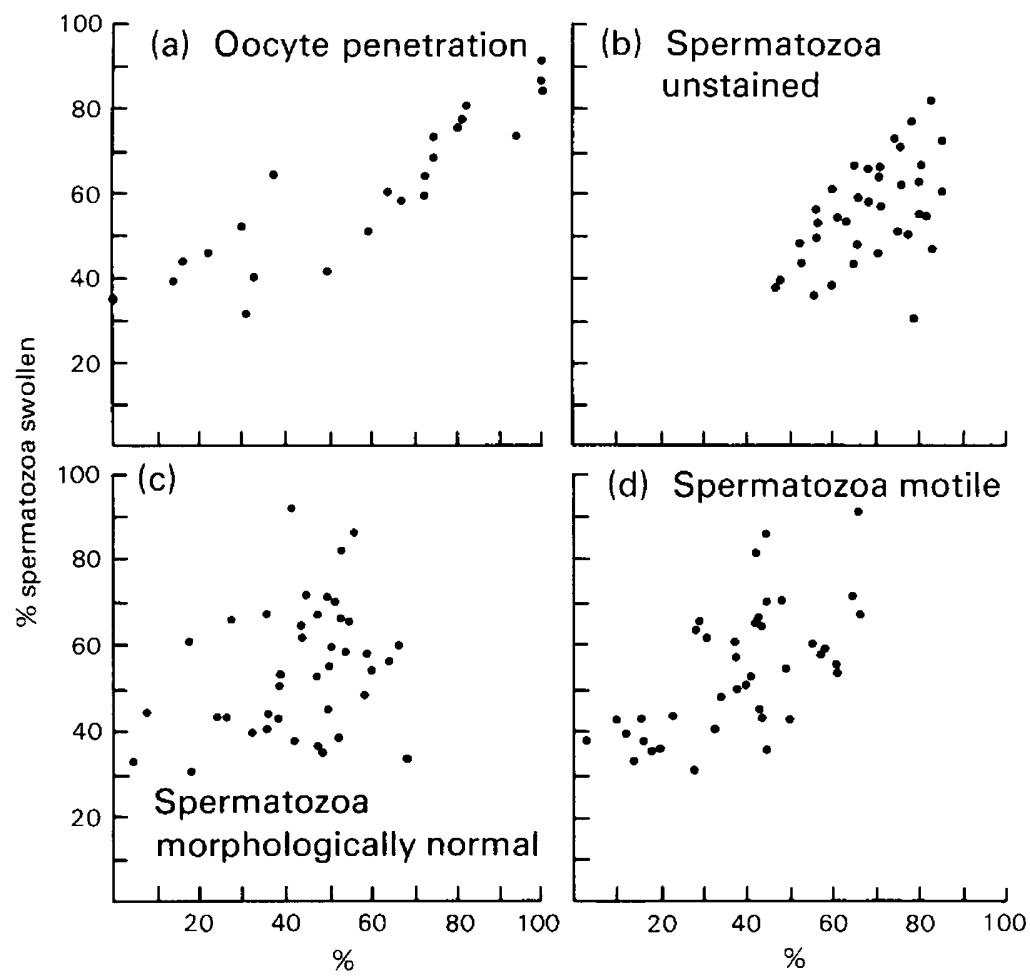

Text-fig. 3. Relationship between the \% of spermatozoa from different semen samples that show swelling under hypo-osmotic stress and (a) the in-vitro fertilizing ability with denuded hamster oocytes $(r=0.90 ; n=23) ;$ (b) the \% spermatozoa not reacting with eosin-Y $(r=0.52$; $n=38)$; (c) the \% spermatozoa with normal morphology $(r=0.30 ; n=40)$; and (d) the $\%$ spermatozoa with forward progressive movements $(r=0.61 ; n=40)$. Different semen samples were used in (a), (b) and (c, d) but the same semen samples in (c) and (d).

penetration, the correlation between the percentage of swollen spermatozoa and motile spermatozoa was greater at a lower percentage of sperm swelling $(<50 \%)$ than at a higher percentage of swelling $(>50 \%)$.

\section{Discussion}

The development of relatively simple assays that can assess the functional activity of spermatozoa should be of use in the diagnosis of the infertile male. Present day semen analyses primarily determine sperm characteristics such as count, motility, forward progression, morphology and agglutination. Although these are representative of the characteristics of spermatozoa, they give inadequate information about the fertilizing capacity of the spermatozoa, i.e., their ability to undergo capacitation, the acrosome reaction and penetration through the oocyte's investments. With the exception of the fructose, citric acid and acid phosphatase levels in seminal plasma, little attention has been given clinically to the chemistry of the ejaculate and particularly that of the spermatozoa. This is in spite of the fact that a number of biochemical events are associated with the male gametes during the reproductive processes. Without their satisfactory completion, the spermatozoa would be incapable of successful fertilization. One of the more important biochemical properties of the spermatozoon is the ability of compounds to be transported across its membranes. 
This is not only essential for the maintenance of sperm motility, but also for the induction of the acrosome reaction and possibly other events related to fertilization. Therefore, assessment of this functional aspect of spermatozoa could be a useful addition to the semen analysis.

The ability of spermatozoa to swell when subjected to hypo-osmotic conditions implies intact membrane function, i.e. the ability of the membrane to allow passage of water in order to establish an equilibrium between the fluid compartment within the spermatozoon and the external surroundings. Evidence that the membrane function measured by this technique is important in the fertilization process is the good correlation $(r=0.90)$ between the percentage of spermatozoa in an ejaculate that are capable of undergoing swelling, and their ability to undergo capacitation and fuse with denuded hamster oocytes. By contrast, no significant correlation was reported between hamster oocyte penetration and: (1) sperm concentration; (2) sperm morphology; (3) sperm motility; (4) number of live spermatozoa; and (5) semen volume (Hall, 1981; Hall, Sloan \& Brown, 1981 ; Cohen, Mooyaart, Vreeburg \& Zeilmaker, 1982), although Rogers et al. (1983) reported that some correlation was present with sperm morphology. Similar comparisons cannot be made in the present study because different semen samples were used for the human sperm-hamster oocyte test and the other sperm assays. However, the present results do show a much smaller correlation between sperm swelling and sperm morphology, sperm motility, and number of live spermatozoa, than that seen between sperm swelling and oocyte penetration. Evidently, sperm swelling is a much better endpoint to assess the ability of spermatozoa to become capacitated and penetrate hamster oocytes than the standard seminal measurements. Although some argument is still present in regard to the human sperm-hamster oocyte test, most investigators agree that the test generally reflects the fertilizing potential of human spermatozoa (Rogers et al., 1979; Overstreet et al., 1980; Hall, 1981; Stenchever et al., 1982). If this is indeed the case, the ability of spermatozoa to swell in a hypoosmotic solution may be a worthwhile addition to the standard semen analysis, particularly since this test is much easier to perform than the human sperm-hamster oocyte assay. Further evaluation of the sperm swelling test from a clinical standpoint is certainly indicated by the present data.

The membrane swelling was particularly noticeable at the sperm tail. Either the sperm tail membrane is more pliant and/or more loosely attached to the underlying structures than the membrane of the sperm head, or only a small fluid compartment is present in the sperm head so that often no observable increase in volume occurs with the influx of water. Although the membranes of the sperm tail and sperm head may function independently from each other, i.e. measuring sperm tail swelling may not be indicative of normal functional activity of the head membranes, this is apparently not usually the case. Capacitation and fusion with the oocyte involves many changes in the sperm head without which these processes cannot occur. Since a good correlation was present between these events and the ability of sperm tails to swell in a hypo-osmotic solution, it is clear that sperm swelling generally indicates normal head membrane function as well. Some exceptions will certainly be present, however, and it must be accepted that the presence or absence of sperm tail swelling will not always be indicative that the sperm head membranes will react normally during capacitation and acrosome reaction. In preliminary studies $(n=7)$ only a $1-18 \%$ variation was observed in the ability of human spermatozoa to swell before and after being capacitated in vitro.

Sperm swelling is only poorly related to the morphological appearance of the spermatozoa, at least as seen after histological staining $(r=0.30)$. It is not surprising that a higher correlation is present between sperm swelling and progressive sperm motility $(r=0.61)$ than between sperm swelling and sperm morphology, because the motility of the spermatozoon also depends in part on the transport of compounds across its membrane. Different characteristics are being measured, however. Sperm swelling is only indicative of normal membrane integrity and function, whereas sperm motility depends not only on membrane transport but also on a large number of other biochemical functions such as sperm metabolism and the microtubular action of the tail fibres. This may explain, at least in part, why the correlation between sperm swelling and motility was greater when the percentage sperm swelling was $<50 \%$. At low percentage swelling, sperm motility would certainly be impaired but at a high percentage swelling, sperm motility may or may not be normal. 
It is also not surprising that the correlation between sperm swelling and 'live' spermatozoa (assessed by the ability of eosin-Y to stain the spermatozoa) was higher than that found between sperm morphology and sperm swelling. The death of spermatozoa results in membrane changes so that eosin-Y can pass. These same membranes would most probably not swell in a hypo-osmotic solution. Different entities are measured, however, because a live spermatozoon may have a nonfunctional membrane, incapable of adjusting to hypo-osmotic stress but still capable of preventing eosin-Y from entering. Apparently, this is quite often the case, otherwise a higher correlation would have been found.

A number of different solutions were tested for their ability to induce swelling of the spermatozoa. A solution containing fructose and sodium citrate at $150 \mathrm{mosmol}$ and a calculated ionic strength of 0.15 gave optimal and repeatable results. It is not known why, at the same osmolality, solutions affect the sperm membranes differently, but presumably sugars and electrolytes have a different influence on the influx of water through the sperm membrane. The spermatozoa showed swelling at $300 \mathrm{mosmol}$, the approximate osmolality of media used for in-vitro fertilization (Miyamoto \& Chang, 1973; Mahi \& Yanagimachi, 1973) and swelling at this osmolality was particularly noticeable in the sucrose and fructose solutions. Since compounds appear to have a different effect on the ability of spermatozoa to swell in a hypo-osmotic solution, it may be worthwhile to screen a number of other solutions besides those used in the present study to determine whether they produce better or even more consistent results. Additionally, it may be advantageous to eliminate from an in-vitro fertilization medium any compounds that significantly enhance sperm swelling at the osmolality of the medium.

We thank Christian Schelthoff for her expert assistance in the preparation of this manuscript. This work was supported by NIH grant HD 09868 and a grant from the Institute of Reproductive Medicine, Chicago, Illinois.

\section{References}

Binor, Z., Sokoloski, J.E. \& Wolf, P.P. (1980) Penetration of the zona-free hamster egg by human sperm. Fert. Steril. 33, 321-327.

Bredderman, P.J. \& Foote, R.H. (1969) Volume of stressed bull spermatozoa and protoplasmic droplets, and the relationship of cell size to motility and fertility. J. Anim. Sci. 28, 496-501.

Cohen, J., Mooyaart, M., Vreeburg, J.T.M. \& Zeilmaker, G.H. (1982) Fertilization of hamster ova by human spermatozoa in relation to semen parameters. Int. J. Androl. 5, 210-224.

Drevius, L.O. (1972) The permeability of bull spermatozoa to water, polyhydric alcohols and univalent anions upon the kinetic activity of spermatozoa and sperm models. J. Reprod. Fert. 28, 41-54.

Drevius, L.O. \& Eriksson, H. (1966) Osmotic swelling of mammalian spermatozoa. Expl Cell Res. 42, 136-156.

Eliasson, R. \& Treichl, L. (1971) Supravital staining of human spermatozoa. Fert. Steril. 22, 134-137.

Foote, R.H. \& Bredderman, P.J. (1969) Sizing of aging bull spermatozoa with an electronic counter. J. Dairy Sci. 52, 117-120.

Hall, J.L. (1981) Relationship between semen quality and human sperm penetration of zona-free hamster ova. Fert. Steril. 35, 457-463.

Hall, J.L., Sloan, C.S. \& Brown, K.G. (1981) Correlations of seminal testosterone and fructose with sperm fertilizing capacity and standard parameters of human semen. J. Androl. 2, 14.

Mahi, C.A. \& Yanagimachi, R. (1973) The effects of temperature, osmolarity and hydrogen ion concentration on the activation and acrosome reaction of golden hamster spermatozoa. J. Reprod. Fert. 35, 5566.

Miyamoto, H. \& Chang, M.C. (1973) The importance of serum albumin and metabolic intermediates for capacitation of spermatozoa and fertilization of mouse eggs in vitro. J. Reprod. Fert. 32, 193-205.

Overstreet, J.W., Yanagimachi, R., Katz, D.F., Hayashi, K. \& Hanson, F.W. (1980) Penetration of human spermatozoa into the human zona pellucida and the zona-free hamster egg: a study of fertile donors and infertile patients. Fert. Steril. 33, 534-542.

Rogers, B.J., Campen, H.V., Neno, M., Lambert, H., Bronson, R. \& Hale, R. (1979) Analysis of human spermatozoal fertilizing ability using zona-free ova. Fert. Steril. 32, 664-670.

Rogers, B.J., Bentwood, B.J., Van Campen, H., Helmbrecht, G., Soderdahl, D. \& Hale, R. (1983) Sperm morphology assessment as an indication of human fertilizing capacity. J. Androl. 4, 119-125.

Stenchever, M.A., Spadoni, L.R., Smith, W.D., Karp, L.E., Shy, K.K., Moore, D.E. \& Berger, R. (1982) Benefits of the sperm (hamster ova) penetration assay 
in the evaluation of the infertile couple. Am. J. Obstet. Gynec. 143, 91-98.

Van der Ven, H.H., Bhattacharyya, A.K., Binor, Z., Leto, S. \& Zaneveld, L.J.D. (1982) Inhibition of human sperm capacitation by a high molecular weight factor from human seminal plasma. Fert. Steril. 38, 753755.
Zaneveld, L.J.D. \& Polakoski, K.L. (1977) Collection and physical examination of the ejaculate. In Techniques of Human Andrology, pp. 147-172. Ed. E. S. E. Hafez. Elsevier/North Holland.

Received 24 May 1983 